\title{
Concept Representation with Overlapping Feature Intervals.
}

Article in Cybernetics and Systems · April 1998

DOI: $10.1080 / 019697298125713 \cdot$ Source: DBLP

CITATIONS

7

2 authors, including:

\section{Halil Altay Güvenir}

Bilkent University

104 PUBLICATIONS 1,515 CITATIONS

SEE PROFILE
READS

16

Some of the authors of this publication are also working on these related projects:

Project

VFCC: voting features based classifier with feature construction, View project

Project

M.S. Thesis at Bilkent University View project 


\title{
CONCEPT REPRESENTATION WTH OVERLAPPING FEATURE INTERVALS
}

\section{H. ALTAY GUVENIR \\ Department of Computer Engineering and Information Science, Bilkent University, Ankara, Turkey}

\section{HAKIME G. KOC}

\author{
Aselsan, Ankara, Turkey
}

\begin{abstract}
This article presents a new form of exemplar-based learning method, based on overlapping feature intervals. In this model, a concept is represented by a collection of overlappling intervals for each feature and class. Classification with Overlapping Feature Intervals (COFI) is a particular implementation of this technique. In this incremental, inductive, and supervised learning method, the basic unit of the representation is an interval. The COFI algorithm learns the projections of the intervals in each feature dimension for each class. Initially, an interval is a point on a feature-class dimension; then it can be expanded through generalization. No specialization of intervals is done on feature-class dimensions by this algorithm. Classification in the COFI algorithm is based on a majority voting among the local predictions that are made individually by each feature. An evaluation of COFI and its comparison with similar other classification techniques is given.
\end{abstract}

Learning refers to a wide spectrum of situations in which a learner increases his knowledge or skill in accomplishing certain tasks. The learner applies inferences to some material in order to construct an appropriate representation of some relevant aspect of reality. The

Address correspondence to Prof. H. Altay Güvenir, Department of Computer Engineering and Information Science, Bilkent University, 06533 Ankara, Turkey. E-mail: guvenir@cs.bilkent.edu.tr

Cybernetics and Systems: An International Journal, 29:263-282, 1998 
process of constructing such a representation is a crucial step in any form of learning.

One of the central insights of AI is that intelligence involves search and that effective search is constrained by domain-specific knowledge. In this framework, machine learning researchers are exploring a vast space of possible learning methods, searching for techniques with useful characteristics and looking for relations between these methods.

Learning from examples has been one of the primary paradigms of machine learning research since the early days of AI. Many researchers have observed and documented the fact that human problem-solving performance improves with experience. In some domains, the principle source of expertise seems to be a memory for a large number of important examples. Attempts to build an intelligent (i.e., at the level of human) system have often faced the problem of memory for too many specific patterns. Researchers expect to solve this difficulty by building machines that can learn using limited resources. This reasoning has motivate d many machine learning projects (Rendell, 1986).

Inducing a general concept description from examples and counterexamples is one of the most widely studied methods for symbolic learning. The goal is to develop a description of a concept from which all previous positive instances can be derived while none of the previous negative instances can be rederived by the same process of rederivation of positive instances. Classification systems require only a minimal domain the ory and they are based on the training instances to learn an appropriate classification function.

In this paper, we propose a new symbolic model for concept learning, based on the representation of overlapping feature intervals (OFIs). The Classification with Overlapping Feature Intervals (COFI, for short) algorithm is a particular implementation of this technique. Feature intervals are formed by generalizing the feature values of training instances from the same class. Overlapping concept descriptions are allowed; that is, there may exist different classes for the same feature values. However, no specialization is done on the concept descriptions.

In the overlapping feature intervals technique, the basic unit of the representation is an interval. Each interval is represented by four parameters: lower and upper bounds, the representativeness count, which is the number of instances that the interval represents, and the associated class of the interval. The intervals are constructed separately 
for each feature dimension and for each class, called feature-class dimension. The construction is through an incremental generalization from the set of training instances. Initially, an interval is a point; that is, its lower and upper bounds are equal to initial feature values of the first training instance for each feature. Then a point interval can be extended to a range interval such that its lower and upper bounds are not equal. This process is based on generalization through close interval heuristic proposed by Winston (1984). Therefore, the description of a concept (class) is the collection of intervals formed on each feature for that class.

Generalization is the main process of training in the COFI algorithm, because it does not use any specilization heuristic. In order to avoid overgeneralization of intervals, generalization is limited with the use of a user-specific parameter. Generalization of an interval to include a new training instance depends on that single external variable, called generalization ratio, and the maximum and the minimum feature value up to current training example. By using this generalization ratio and the se local maximum and minimum feature values, a generalization distance is calculated. Whether a feature is joined to an existing interval or constructs a separate point interval is determined by this generalization distance. Small generalization ratios cause a large number of small intervals to be constructed, whereas large generalization ratios cause a small number of large intervals.

Classification in COFI is simply a search for the intervals corresponding to the value of the test instance for each feature. A feature votes for the classes of such intervals. The vote of a feature for a class is the relative representativeness count, which is the ratio of the representative ness count of the matched interval to the total number of training instances of that class. The votes of a feature are maintaine $d$ in a vote vector, whose elements represent the votes for each class. For the final classification of a test instance, the vote vectors of features are summed. The class that received the highest amount of votes is declared to be the class of the test instance.

The COFI algorithm handles unknown attribute and class values in a straightforward manner. Similarly to human behavior, it just ignores these unknown attribute and class values. Most of the learning systems usually overcome this problem by either filling in missing values with the most probable value or a value determined by exploiting interrela- 
tionships among the values of different attributes or by looking at the probability distribution of known feature values (Quinlan, 1993).

In the next section, we will present some of the existing concept learning models. Then a detailed explanation of our new algorithm, COFI, will be given. Later, we will evaluate the COFI algorithm by giving the complexity analysis and the results of empirical evaluation on some real-world datasets.

\section{EXEMPLAR-BASED MODELS}

Exemplar-base d learning is a kind of concept learning methodology in which the concept definition is constructed from the examples themselves, using the same representation language. There are two main types of exemplar-based learning methodologies in the literature, namely instance-based learning and exemplar-based generalization (see Figure 1). Instance-based learning retains examples in memory as points in feature space and never changes their representation. However, in examplar-based generalization techniques the point-storage model is slightly modified to support generalization.

An instance-based concept description includes a set of stored instances along with some information concerning their past performance during the training process. The similarity and classification functions determine how the set of saved instances in the concept description is used to predict values for the category attribute. There-

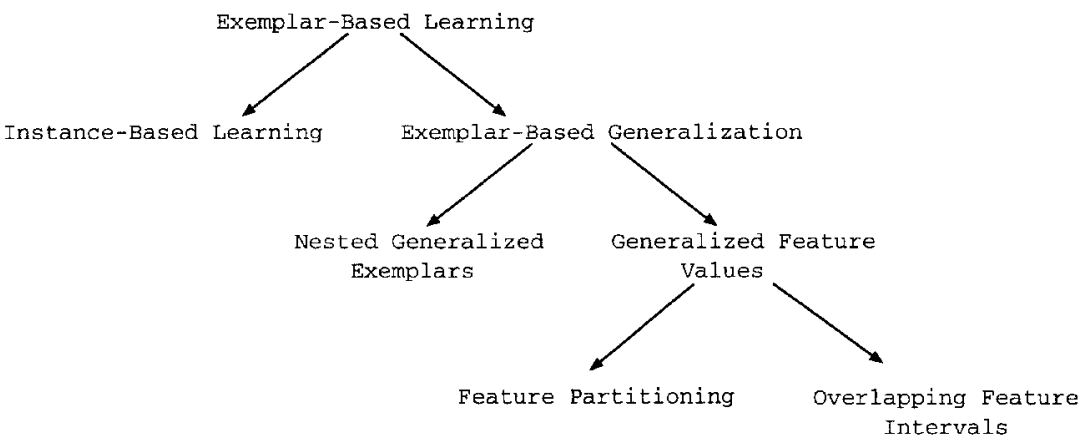

Figure 1. Exemplar-based learning algorithms. 
fore, IBL concept descriptions contain these two functions along with the set of stored instances.

The instance-based learning technique (Aha et al., 1991) was first implemented in three different algorithms, namely IB1, IB2, and IB3. IB 1 stores all the training instances; IB2 stores only the instances for which the prediction was wrong. Neither IB1 nor IB2 removes any instance from concept description after it had been stored. IB3 is the noise-tole rant version of the IB2 algorithm. It employs a significance test to determine which instances are good classifiers and which ones are believed to be noisy. Later Aha (1992) implemented two extensions to the se algorithms, called IB4 and IB5. IB4 learns a separate set of attribute weights for each concept, which are then used in the similarity function. IB5, which is an extension of IB4, can cope with novel attributes by updating an attribute's weight only when its value is known for both the instance being classified and the instance chosen to classify it.

IBL algorithms assume that instances that have high similarity values according to the similarity function have similar classifications. This leads to their local bias for classifying novel instances according to their most similar neighbor's classification. They also assume that without prior knowledge attributes will have equal relevance for classification decisions (i.e., each feature has equal weight in the similarity function). This assumption may lead to significant performance degradation if the data set contains many irrelevant features.

In Nested Generalized Exemplars (NGE) the ory, learning is accomplished by storing objects in Euclidean $n$-space, $E^{n}$, as hyper-rectangle s (Salzberg, 1991). NGE adds generalization on top of the simple exemplar-base d learning. It adopts the position that exemplars, once stored, should be generalized. The learner compares a new example to those it has seen before and finds the most similar, according to a similarity metric that is inversely related to the distance metric (Euclidean distance in $n$-space). The term exemplar (or hyperrectangle) is used to denote an example stored in memory. Over time, exemplars may be enlarged by generalization. Once a theory moves from a symbolic space to Euclidean space, it becomes possible to nest one generalization inside the other. Its generalizations, which take the form of hyperrectangles in $E^{n}$, can be nested to an arbitrary depth, where inner rectangles act as exceptions to the outer ones. 
EACH (Exemplar-Aided Constructor of Hyperrectangles) is a particular implementation of the NGE technique, in which an exemplar is represented by a hyperrectangular. EACH uses numeric slots for feature values of every exemplar. The generalizations in EACH take the form of hyperrectangles in Euclidean $n$-space, where the space is defined by the feature values for each example. Therefore, the generalization process simply replaces the slot values with more general values (i.e., replacing the range $[a, b \rrbracket$ with another range $\llbracket c, d]$, where $c \leqslant a$ and $b \leqslant d$ ). EACH compares the class of a new example with the most similar (shortest distance) exemplar in memory. The distance between an example and an exemplar is computed similarly to the similarity function of IBL algorithms, but exemplars and features also have weights in this computation and the result is the distance.

In the feature partitioning (FP) techniques, examples are stored as partitions on the feature dimensions. One example of the implementation of feature partitioning is the Classification by Feature Partitioning (CFP) algorithm of Güvenir and Şirin (1996). Learning in CFP is accomplished by storing the objects separately in each feature dimension as disjoint sets of values called segments. A segment is expanded through generalization or specialized by dividing it into subsegments. Classification is based on a weighted voting among the individual predictions of the features, which are simply the class values of the segments corresponding to the values of a test instance for each feature. Descriptions of the same concepts in both FP and OFI are presented in Figure 2. The main difference between FP and OFI techniques is that FP uses disjoint partitioning of feature values into nonoverlapping intervals (segments), whereas OFI allows overlapping of intervals that belong to different classes.

\section{THE COFI ALGORITHM}

The input of the COFI algorithm is a training data set, and the output is the classification knowledge in the form of overlapping feature intervals. Each instance in the training data set is represented by a vector. The $i$ th instance $e_{i}$ is represented as $e_{i}=\left\langle x_{i, 1}, x_{i, 2}, \ldots, x_{i, n}, c_{j}\right\rangle$ where $x_{i, 1}, x_{i, 2}, \ldots, x_{i, n}$ are the corresponding feature values of features $f_{1}, f_{2}, \ldots, f_{n}$ and $c_{j}$ is the associate $d$ class of the example $e_{i}$, where $1 \leqslant j \leqslant k$, and $k$ is the total number of the classes. 


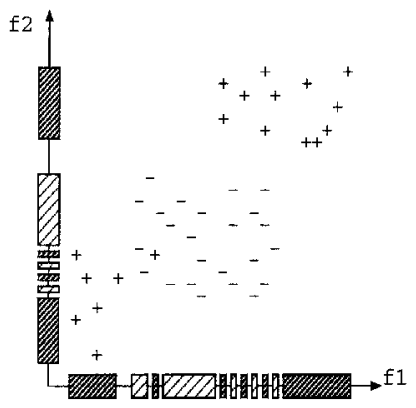

(a)

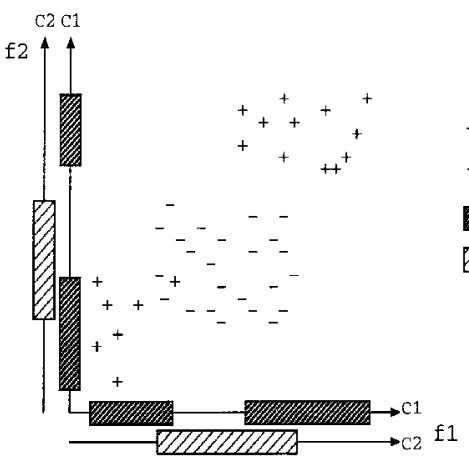

$+\mathrm{Cl}$ training instance

- C2 training instance

C1 interval $\oslash \mathrm{C} 2$ interval

(b)

Figure 2. Comparison of representation by feature partitioning and overlapping feature intervals for the same data set.

\section{Learning in COFI}

Learning overlapping feature intervals is done by storing the projections of objects separately on each feature-class dimension and then generalizing these values of the same class into intervals. The basis unit of the representation is an interval. An interval is represented by its lower and upper bounds, representative ness count, and its class.

$I=\langle[$ lower bound, upper bound $]$, class, representativeness count $\rangle$

Lower and upper bounds of an interval are the minimum and maximum feature values that fall into the interval, respectively. Representative- 
ness count is the number of the instances that the interval represents, that is, the instances that have the corresponding feature value between lower and upper bounds, and their class value is the same as the class value of the interval.

In the COFI algorithm, learning is achieved by obtaining the projection of the concepts over each class dimension for each feature. Initially, when the first example is processed, a point interval is constructed. The lower and upper bounds of this interval are equal to the corresponding feature value. It is represented as a point in the feature dimension of the corresponding class.

As an example, suppose that the first training instance of a framed data set $e_{1}$ is of class $c_{1}$. As shown in Figure 3a, the corresponding feature-class dimensions are update $\mathrm{d}$ and a point interval is constructe $\mathrm{d}$ for $c_{1}$ on each feature. In other words, if the first example is $e_{1}=$ $\left\langle x_{1,1}, x_{1,2}, c_{1}\right\rangle$ where $x_{1,1}$ and $x_{1,2}$ are the feature values of $f_{1}, f_{2}$ and $c_{1}$ is the associated class, then a point interval will be constructed for the corresponding class dimension of each feature. The point interval first partitions the corresponding class dimension of a feature into three intervals. The first interval's lower bound is $-\infty$, upper bound is $x_{1,1}$, and representative ness count is 0 ; the second interval's lower and upper bounds are $x_{1,1}$ and the representativeness count is 1 ; finally, the third and the last interval's lower bound is $x_{1,1}$, upper bound is $\infty$, and representativeness count is 0 again. The first and third intervals' class value is associated as UNDETERMINED but the second interval's class value is determined by the related instance's class value, $c_{1}$. Therefore, the intervals for feature $f_{1}$ in Figure $3 \mathrm{a}$ are represented as follows:

$$
\begin{aligned}
& \left\langle\left(-\infty, x_{1,1}\right), \text { UNDETERMINED, } 0\right\rangle \\
& \left\langle\left[x_{1,1}, x_{1,1}\right], c_{1}, 1\right\rangle \\
& \left\langle\left(x_{1,1}, \infty\right), \text { UNDETERMINED }, 0\right\rangle
\end{aligned}
$$

For the second feature $f_{2}$, a similar representation is constructed. If the next training example belongs to another class, the same procedure is applied and a new point interval is formed in this new corresponding class dimension. However, if it belongs to the same class, then there is a potential for generalization of intervals. An interval may be extended 
(a)

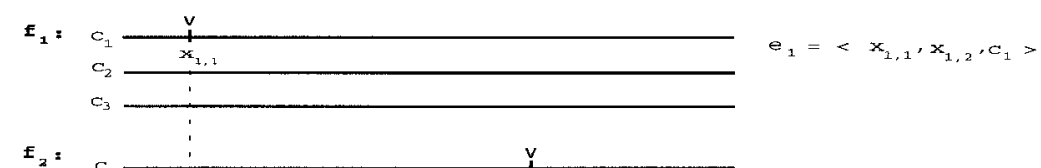

(b)

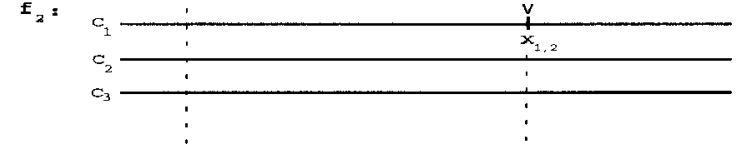

(c)
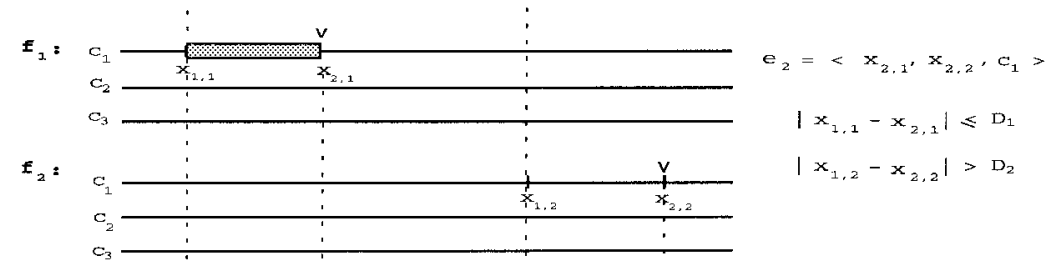

(d)

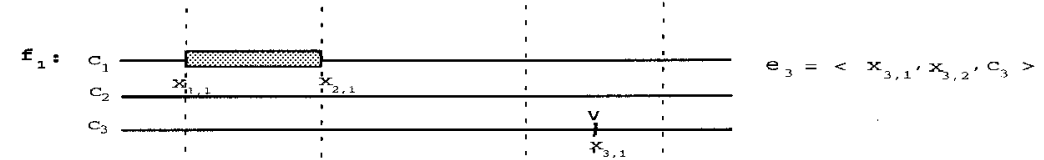

(e)

$\pm_{2}=$
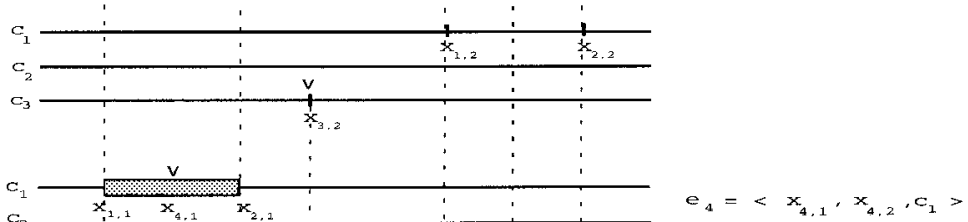

$f_{2}=$

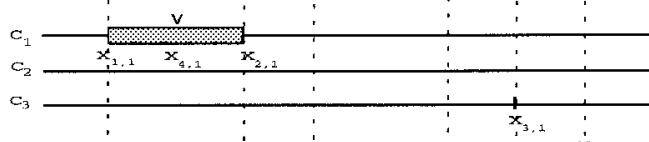

$e_{4}=\left\langle x_{4,1}, x_{4,2}, c_{1}\right\rangle$

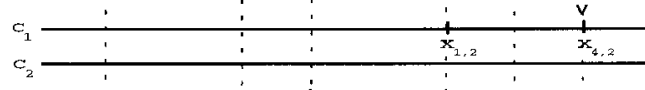

$x_{4,2}=x_{2,2}$

$c_{3}$

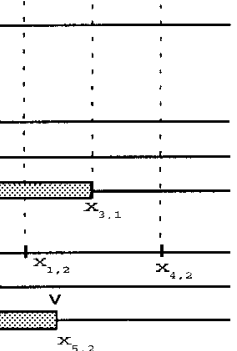

$e_{5}=\left\langle x_{5,1}, x_{5,2}, c_{3}\right\rangle$

$\left|x_{5,1}-x_{3,1}\right| \leqslant D_{1}$

$\left|x_{3,2}-x_{5,2}\right| \leqslant D_{2}$

Figure 3. An example of the construction of the intervals in the COFI algorithm.

through generalization with other neighboring points of intervals in the same class dimension to cover the new feature value. In order to generalize the new feature value, the distance between this value and the previously constructe $d$ intervals must be less than the generalization distance, $D_{f}$, for this feature. The generalization distance $D_{f}$ for each 
feature is determined dynamically as

$$
D_{f}=\left(\text { current }-\max _{f}-\text { current }-\min _{f}\right) \times g
$$

Here, current- $\max _{f}$ and current-min $\max _{f}$ are maximum and minimum values for the feature $f$ seen up to the current training instance, respectively. This range is multiplied by a global parameter $g$, called the generalization ratio. The parameter $g$ is selected in the range $[0,1]$.

The generalization process is applied only to linear features. Therefore, if nominal feature values are processed then no generalization is done and $D_{f}$ values are taken as 0 for these kinds of features. If the distance between the current training instance and the previously constructed intervals is greater than the $D_{f}$, then the training instance constructs a new point interval. If, on the other hand, the training instance falls properly in an interval, then the representative ness count of that interval is simply incremented by one.

Suppose that the $f_{1}$ value of the second training instance $e_{2}$ is $x_{2,1}$ and the distance between $x_{2,1}$ and the point interval $\left\langle\left[x_{1,1}, x_{1,1}\right], c_{1}, 1\right\rangle$ is less than the generalization distance $D_{1}$. Also suppose that the $f_{2}$ value of the second instance $e_{2}$ is $x_{2,2}$ and the distance between $x_{2,2}$ and the point interval $\left\langle\left[x_{1,2}, x_{1,2}\right], c_{1} 1\right\rangle$ is greater than the generalization distance $D_{2}$. That is, for the second example $e_{2}=\left\langle x_{2,1}, x_{2,2} c_{1}\right\rangle$,

$\left|x_{1,1}-x_{2,1}\right| \leqslant D_{1} \quad$ and $\quad\left|x_{1,2}-x_{2,2}\right|>D_{2}$

For the first feature $f_{1}$, generalization will occur on the $c_{1}$ dimension of $f_{1}$, but no generalization will be done on $f_{2}$,s class dimension $c_{1}$. The generalization process is illustrated in Figure $3 \mathrm{~b}$. The COFI algorithm will generalize the interval for $x_{1,1}$ into an extended interval $\left\langle\left[x_{1,1}, x_{2,1}\right], c_{1}, 2\right\rangle$. Here, the representativeness count is also incremented by one, because this interval represents two examples now. Another point interval is constructed in $f_{2}$ 's corresponding class dimension, so the $c_{1}$ dimension of this feature will have two point intervals $\left\langle\left[x_{1,2}, x_{1,2}\right], c_{1}, 1\right\rangle$ and $\left\langle\left[x_{2,2}, x_{2,2}\right], c_{1}, 1\right\rangle$, along with three interleaving range intervals of UNDETERMINED class.

If the new training example falls into an interval with a different class, then the same procedures are executed for this new class dimension. If, for example, third training instance $e_{3}$ 's class is $c_{3}$, then the partitioning will be done in $c_{3}$ 's class dimension as in Figure $3 \mathrm{c}$. This 
property of the algorithm allows overlapping, because at the same time, different class intervals may be formed for the same feature values.

If one of the feature values of the next training example falls into an interval properly as shown in Figure 3d, then the representative ness count of the interval is incremented by one; that is, if the interval is $\left\langle\left[x_{1,1}, x_{2,1}\right], c_{1}, 2\right\rangle$ and the next instance's $f_{1}, x_{3,1}$, is between $x_{1,1}$ and $x_{2,1}$, that is, $x_{1,1} \leqslant x_{3,1} \leqslant x_{2,1}$, then the new interval will be $\left.\left\langle x_{1,1}, x_{2,1}\right], c_{1}, 3\right\rangle$. No physical change occurs on the boundaries of the interval but the representativeness count is incremented by one.

Perhaps the most importance characteristic of the COFI algorithm is that it allows overlapping of intervals that are generalizations of feature values. That is, it has the ability to form different class or concept definitions for the same feature values. The rationale behind this approach is based on the fact that there may exist different class values for the overlapping feature values. The COFI algorithm may store many intervals that correspond to the same feature values but different class values. Here it is assumed that there are disjunctive concepts.

Let us show the formation of overlapping intervals through an example. Let the fifth training instance of Figure 3 belong to $c_{3}$. The feature values of this instance are shown in Figure 3c. Assume that the generalization distance $D_{1}$ is greater than the distance between $x_{3,1}$ and $x_{5,1}$, so a range interval is constructe $\mathrm{d}$ for the $c_{3}$ class dimension of $f_{1}$. Similarly, $D_{2}$ is greater than the distance between $x_{3,2}$ and $x_{5,2}$, and again a range interval is constructed for the second feature's $c_{3}$ class dimension. Here, the ove rlap occurs between the descriptions of class $c_{1}$ and $c_{3}$. For the feature $f_{1}$, overlap occurs between the feature values $x_{5,1}$ and $x_{2,1}$, and for the feature $f_{2}$ overlap occurs at the point $x_{1,2}$. In Figure $3 \mathrm{e}$, it is seen that in the COFI algorithm no specialization is done, that is, there is no subdivision of existing intervals. This approach is plausible, because in real life different concepts may exist at the same time, especially in medical domains.

\section{Classification in COFI}

The classification of a test instance is based on a majority voting taken among the individual predictions based on the votes of the features. The vote of a feature is based solely on the value of the test instance for that feature. The vote of a feature is not for a single class but rather a 
vector of votes, called a vote vector. The size of the vector is equal to the number of classes. An element of the vote represents the vote that is given by the feature to the corresponding class. The vote that a feature gives to a class is the relative representativeness count of the class interval. The relative reprsentative ness count is the ratio of the representativeness count to the number of examples of the corresponding class value. Because most of the data sets are not distributed normally in terms of their class values, this kind of normalization is required. The vote vectors of each feature are added to determine the predicted class. The class that receives the maximum vote is the final class prediction for the test instance.

For a given test instance $i$, the feature value $i_{f}$ is searched on all class dimensions for feature $f$. The results of the search for feature $f$ are summarized in a vote vector

$\mathbf{v}_{f}=\left\langle v_{f, 1}, v_{f, 2}, \ldots, v_{f, k}\right\rangle$

Here, $k$ is the number of classes and

$v_{f, c}=\left\{\begin{array}{l}\text { relative representiveness count of } I \\ \text { if } i_{f} \text { falls in interval } I \text { of class } c \text { on feature } f \\ 0 \quad \text { otherwise }\end{array}\right.$

The final vote vector $\mathbf{v}$ is obtaine $\mathrm{d}$ by summing the se vectors:

$\mathbf{v}=\sum_{f=1}^{n} \mathbf{v}_{f}=\left\langle v_{1}, v_{2}, \ldots, v_{c}, \ldots, v_{k}\right\rangle$

where $v_{c}=\sum_{f=1}^{n} v_{f, c}$.

The final classification for the test instance $i$ is the class that received the highest amount of votes:

classification $(i)=c$, such that $v_{c}>v_{j}$ for each $j \neq c$

If the feature value $i_{f}$ does not fall into any interval in any class dimension, then the class is predicted as UNDETERMINED and no votes (in other words, 0 votes) are assigned to the vote vector elements. That is, no prediction is made for this value on that feature.

As an example of the classification, suppose that the intervals are formed for all the training set elements as shown in Figure 4. For 


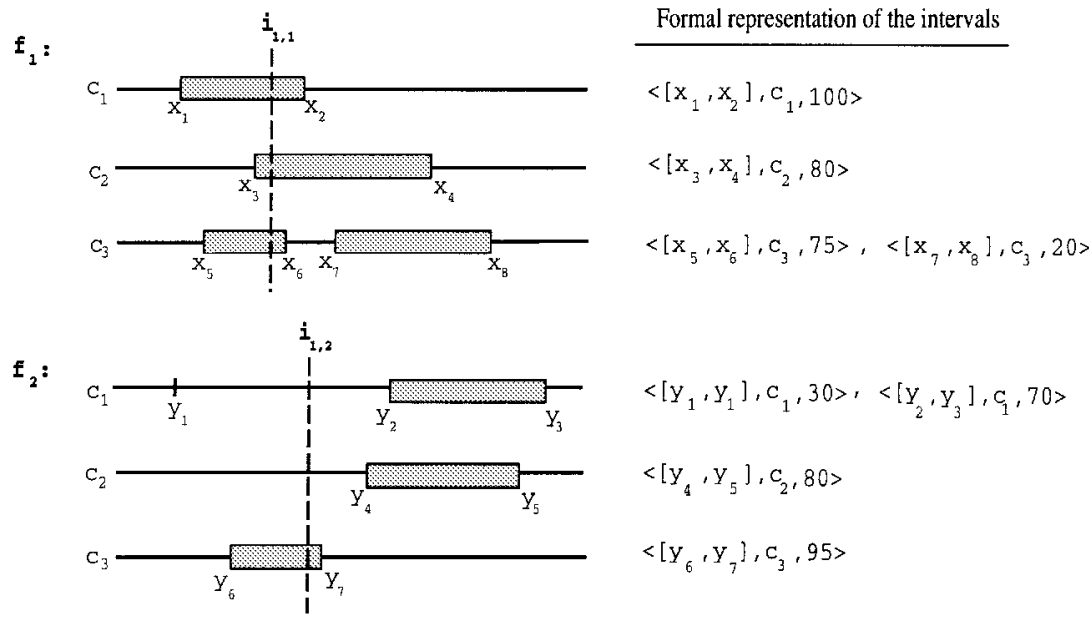

Figure 4. An example of classification in the COFI algorithm.

feature $f_{1}$, there exist one interval for $c_{1}$ and $c_{2}$ and two intervals for class $c_{3}$. The corresponding intervals are also shown in Figure 4. For the second feature $f_{2}$, there exist two intervals for $c_{1}$, and one of them is a point interval. There is one interval for each of $c_{2}$ and $c_{3}$.

In determining the vote of a feature for a class, there are two possibilities. A test instance $i$ may fall into an interval or not. Let the test instance $i$ 's first and second feature values $i_{1,1}$ and $i_{1,2}$ be given as shown in Figure 4. To form the vote vectors, first the relative representativeness count of each matched interval should be computed. As seen in Figure 4, the class counts of $c_{1}, c_{2}$, and $c_{3}$ are 100, 80, and 95, respectively.

For this case, the vote vector of the first feature is

$\mathbf{v}_{1}=\langle 100 / 100,80 / 80,75 / 95\rangle=\langle 1,1,0.79\rangle$

and the vote vector $\mathbf{v}_{2}$ of the second feature is

$\mathbf{v}_{2}=\langle 0 / 100,0 / 80,95 / 95\rangle=\langle 0,0,1\rangle$

The vote vectors of the features are added to reach a final prediction, and then the resulting vector $\mathbf{v}$ is

$\mathbf{v}=\mathbf{v}_{1}+\mathbf{v}_{2}=\langle 1,1,0.79\rangle+\langle 0,0,1\rangle=\langle 1,1,1.79\rangle$ 
Because the vote that class $c_{3}$ receives is the highest, the final classification is the class $c_{3}$.

\section{EVALUATION OF COFI}

In this section, both a complexity analysis and an empirical evaluation of the COFI algorithm are presented. In the complexity analysis, space, training time, and testing time complexities of the COFI algorithm are computed. In the empirical evaluation, the COFI algorithm is compared with the $k-\mathrm{NN}$, the $\mathrm{NBC}$, and the CFP algorithms on some real-world data sets.

\section{Complexity Analysis of the COFI Algorithm}

Space complexity of the COFI algorithm is usually less than that of other techniques that store examples verbatim in memory. However, in the worst case, the space complexity of the COFI algorithm is

\section{$O(m n)$}

where $m$ is the total number of training instances and $n$ the total number of features hence $m n$ is the maximum total number of intervals.

The time complexity of the COFI algorithm depends on the number of intervals. The number of intervals is determined by the generalization ratio, the nature of attributes of the training examples, and the repeated feature values of the training examples. For nominal features no generalization is done. In this case, all the intervals are point intervals. In the worst case, if all of them have unique values, then the number of intervals is equal to the number of training examples for each feature. This is a very rare situation in real-world data sets; if it occurs then these kinds of features are usually irrelevant features.

Learning in the COFI algorithm involves the update of the current concept representation for each training instance. Updating the current representation with a new training instance requires a search for the interval in which the training instance falls for each feature-class dimension. In our implementation, intervals are stored in an ordered list, and we use a simple linear search. The complexity of this search is proportional to the number of existing intervals currently on the corre- 
sponding feature class dimension. In the worst case the number of intervals for one feature is $m$. Therefore, the time required by the training process for the $i$ th instance is

$c_{t} \times n \times k \times(i / k)$

where $c_{t}$ is the training constant, $k$ is the number of classes, and $i / k$ is the average number of examples for each class up to the $i$ th training instance. For all the training instances, this time will be

$c_{t} \times m \times n \times k \times(m / k)$

Here, the average number of intervals for one class dimension is $m / k$, because we have $k$ class dimensions for each feature, and there is a minimum of $m$ intervals for each feature. Therefore, the training time complexity of the COFI algorithm is

$O\left(m^{2} n\right)$

The classification of a test instance requires a search on each class dimension for all features. Since, in the worst case, there will be $m$ intervals, the complexity of classification of a test instance is equal to the sequential search time on ordered lists, which is

$O(m n)$

By using an appropriate data structure, for example, balanced binary tree, a search can be done in $O(\log m)$ time. However, for its simplicity in the implementation we choose to maintain the intervals on a feature-class dimension as an ordered list.

\section{Empirical Evaluation of the COFI Algorithm}

The COFI algorithm is tested on some real-world data sets that are widely used in the machine learning field. The real-world data sets are selected from the collection of data sets distributed by the machine learning group at the University of California at Irvine.

We compared the COFI algorithm with the NBC (Naive Bayesian Classifier) (Duda \& Hart, 1973), $k$-NN ( $k$ Nearest Neighbor Classifier), and CFP (Classification by Feature Partitioning) (Güvenir \& Şirin, 1996) algorithms. The results of the comparison are given in Table 1. 
Table 1. Comparison of NBC, $k$-NN, CFP, and COFI in terms of accuracy (\%) and memory (number of values stored) on some real-world data sets using the leave-one-out evaluation technique

\begin{tabular}{|c|c|c|c|c|c|c|c|c|c|}
\hline \multirow[b]{2}{*}{ Data set } & \multicolumn{2}{|c|}{ NBC } & \multicolumn{2}{|c|}{$1-\mathrm{NN}$} & \multicolumn{2}{|c|}{ CFP } & \multicolumn{3}{|c|}{ COFI } \\
\hline & Acc & Mem & Acc & $\mathrm{Mem}$ & Acc & Mem & Acc & Mem & $g$ \\
\hline Iris & 95.33 & 745 & 95.33 & 745 & 96.00 & 606 & 92.67 & 180 & 0.020 \\
\hline Glass & 57.01 & 2130 & 70.09 & 2130 & 53.27 & 5264 & 57.94 & 1098 & 0.014 \\
\hline Horse-colic & 80.70 & 8441 & 79.61 & 8441 & 81.52 & 2792 & 79.89 & 504 & 0.300 \\
\hline Ionosphere & 88.60 & 12250 & 86.89 & 12250 & 89.46 & 20552 & 94.30 & 280 & 0.270 \\
\hline Hungarian & 83.33 & 4102 & 76.53 & 4102 & 81.29 & 1976 & 85.37 & 196 & 0.250 \\
\hline Cleveland & 80.53 & 4228 & 77.56 & 4228 & 84.82 & 2452 & 82.83 & 244 & 0.100 \\
\hline Wine & 93.26 & 2478 & 94.94 & 2478 & 89.89 & 5215 & 96.63 & 164 & 0.200 \\
\hline
\end{tabular}

Our implementation of the NBC algorithm does not make any assumption about the distribution of values for features, such as a normal distribution. Therefore, both $\mathrm{NBC}$ and $k$-NN algorithms store all the instances in the memory.

The CFP algorithm is similar to the COFI algorithm. CFP uses feature partitioning instead of overlapping feature intervals. A set of feature values is partitioned into segments of the same class. Whereas COFI assigns weights to intervals, CFP learns a weight for each feature.

In this paper, the leave-one-out cross-validation technique is used to report the performance of the COFI algorithm. Leave-one-out is an elegant and straightforward technique for estimating classifier error rates. Because it is computationally expensive, it is often reserved for relatively small samples. For a given method and sample size (number of instances) $m$, a classifier is generated using $m-1$ cases and tested on the remaining case. This process is repeated $m$ times and the total number of correct classifications is taken as the leave-one-out crossvalidation accuracy of the learning algorithm on the given data set. The values given for memory sizes in Table 1 are the number of values store d after training with $m-1$ training instances.

It is seen that the COFI algorithm achieves better results than the other algorithms on four out of seven of these data sets. All the classifiers achieve similar accuracies for the Iris, and Horce-colic data sets. The $k$-NN algorithm outperforms the others on the Glass data set; but does poorly on the Cleveland data set. 
The CFP and the COFI algorithm usually achieve similar accuracy results, and they are better than $\mathrm{NBC}$ and $k-\mathrm{NN}$ on the average. The COFI algorithm results in better accuracy for the Glass, Ionosphere, Hungarian, and Wine data sets than the CFP algorithm. It is seen that if the ove rall performance is considered, the COFI algorithm achieves the best accuracy results among these four algorithms for the seven data sets.

When memory requirements among these algorithms are compare $\mathrm{d}$, it is clear that the COFI algorithm always requires less memory than the other algorithms. The number of intervals stored in memory by the COFI algorithm depends on the generalization ratio and the structure of the data set, in other words, the distribution of the feature values. Four values are stored in memory for each interval. On the other hand, in the $\mathrm{NBC}$ and $k$-NN algorithms, all the instances are kept in memory verbatim. Therefore, for each instance the feature values and the associated class value should be stored. Table 1 gives the memory requirements of these algorithms for the seven real-world data sets. Here we assumed that one unit of memory is allocated for each element of an interval and for each feature and class value.

In the CFP algorithm, segments are stored in memory. However, since no overlapping is allowed and subpartitioning is done in the CFP algorithm, usually the number of segments of the CFP algorithm is greater than the number of intervals of the COFI algorithm. Memory requirements of the CFP algorithm are also shown in Table 1.

\section{CONCLUSION}

We have presented a new methodology of learning from examples that is a new form of exemplar-base $d$ generalization technique based on the representation of overlapping feature intervals. This technique is called Classification with Overlapping Feature Intervals (COFI). COFI is an inductive, incremental, and supervised concept learning method. It learns the projections of the intervals in each class dimension for each feature. Those intervals correspond to the learned concepts.

The COFI algorithm is similar to the NBC and CFP algorithms in that all of these techniques process each feature separately. All of them use feature-based representation, and the classification process is based on majority voting for these algorithms. However, COFI requires much less memory than the others, because in the training process $\mathrm{NBC}$ and 
NN keep all examples with all feature and class values in memory separately. However, in COFI, only intervals are stored. Therefore, when compared with many other similar techniques, the COFI algorithm's memory requirement is less than their requirements. A version of the $k$-NN algorithm, called $k$-NNFP (for $k$ Nearest Neighbors on Feature Projections), has also been shown to achieve about the same accuracy as the $k$-NN algorithm (Akkus, \& Güvenir, 1996). The $k$-NNFP algorithm also requires much less memory than the $k$-NN algorithm.

The COFI algorithm is applicable to domains, where each feature can be used in classification independently of the others. Holte (1993) has pointed out that most data sets in the UCI repository are such that, for classification, their features can be considered independently of each other. Also, Kononenko (1993) claimed that in the data used by human experts there are no strong dependences between features because features are properly defined.

An important characteristic of the COFI algorithm is its ability of overlapping. When compared with CFP, COFI is successful when CFP fails in some cases. For example, if the projections of concepts on an axis overlap each other, the CFP algorithm constructs many segments of different classes next to each other. In that case, the accuracy of classification depends on the observed frequency of the concepts. However, in the COFI algorithm, because all class dimensions are independent of each other, no specialization is required. The concept descriptions can be overlapped.

Another important property of the COFI algorithm is its means of handling the unknown attribute values. Most of the systems use ad hoc methods to handle the unknown attribute values (Quinlan, 1989; Grzymala-Busse, 1991). Like CFP, the COFI algorithm also ignores the unknown attribute values, which is a natural and plausible approach. Because the value of each attribute is handled separately, this causes no problem.

The behavior of the COFI algorithm toward the irrelevant features is also very interesting. Irrelevant attributes can easily be detected by looking at the concept description of the COFI algorithm. Irrelevant attributes construct intervals that cover whole dimension for each class. Therefore, the detection of the irrelevant attributes can be performed by looking at the intervals of the COFI algorithm. In the CFP algorithm, irrelevent attributes cause fragmentation of the feature dimensions into a large number of small segments. Whereas the COFI 
algorithm decreases the number of intervals, the CFP algorithm increases its segments of irrelevant features.

The COFI algorithm uses the relative representative ness count for prediction, which is the number of examples that interval represents. This number is divided by the total number of examples that have the same class value. Therefore, a kind of normalization is achieved to make the correct predictions. Such a normalization is needed because datasets usually contain nonhomoge neous examples in terms of the number of examples that have the same class value.

One important component of the COFI algorithm is the generalization ratio $g$. It controls the generalization process. This ratio is chosen externally depending on experiments. For future work, an algorithm may be developed to find the optimum ge neralization ratio.

One of the most important properties of the COFI algorithm is that one may easily predict the class of a given test instance by using the learned concepts. The algorithm does not have to search all features if only an approximate answer suffices. However, in some techniques, for example, in decision trees, the algorithm has to search all features until it reaches a leaf (Utgoff, 1994). In the COFI algorithm, a decision can be made by just looking at some key attributes. This approach is similar to the human approach of classification.

At the end, the simplicity of the rules for the concept descriptions in the COFI algorithm should be expressed. The simplicity of the algorithm does not affect the accuracy results when compared with the very complex algorithm NBC. NBC represents its knowledge in the form of probability distribution functions. Simple-rule learning systems are generally a viable alternative to systems that learn more complex rules by applying more complex algorithms. If a complex rule is induced, then its additional complexity must be justified by giving more accurate predictions than a simple rule.

When compared with the $\mathrm{NBC}$ and $k$-NN algorithms, the COFI algorithm uses much less storage, because both the NBC and $k$-NN algorithms should keep all feature values separately in the memory to find the probability density function in NBC and to find the distance measure in $k$-NN for predictions. In the COFI algorithm, intervals should be kept in memory. The memory required for intervals is usually much less than the memory required for the instances themselves.

In summary, the scheme for knowledge representation in the form of overlapping feature intervals is a viable technique in classification. 
The COFI algorithm can compete with the well-known machine learning algorithms in terms of accuracy. Also, the requirement of much less memory and the high learning and classification speed of the algorithm are its important advantages.

\section{REFERENCES}

Aha, D. W. 1992. Tolerating noisy, irrelevant and novel attributes in instancebased learning algorithms. Int. J. Man-Machine Studies 36:267-287.

Aha, D. W., D. Kibler, and M. K. Albert. 1991. Instance-based learning algorithms. Mach. Learn 6:37-66.

Akkuş, A., and H. A. Güvenir. 1996. $K$ nearest neighbor classification on feature projections. Proceedings International Conference on Machine Learning, ICML'96, ed. L. Saitta, pp. 12-19. Morgan Kaufmann. San Mateo, CA: Italy, July.

Duda, R. D., and P. E. Hart. 1973. Pattern classification and scene analysis, New York: Wiley.

Grzymala-Busse, J. W. 1991. On the unknown attribute values in learning from examples. Proceedings Sixth International Symposium Methodologies for Intelligent Systems, pp. 368-377, October.

Güvenir, H. A., and I. Şirin. 1996. Classification by feature partitioning. Mach. Learn. 23:47-67.

Holte, R. C. 1993. Very simple classification rules perform well on most commonly used datasets. Mach. Learn. 11:63-91.

Kononenko, I. 1993. Inductive and Bayesian learning in medical diagnosis. Appl. Artif. Intell. 7:317-337.

Quinlan, J. R. 1989. Unknown attribute values in induction. Proceedings 16th International Workshop on Machine Learning, ed. A. Segre, pp. 164-168. San Mate o, CA: Morgan Kaufmann.

Quinlan, J. R. 1993. C4.5: Programs for machine learning. San Mateo, CA: Morgan Kaufmann.

Rendell, L. 1986. A general framework for induction and a study of selective induction. Mach. Learn. 1:177-226.

Salzberg, S. 1991. A nearest hyperrectangle learning method. Mach. Learn. 6:251-276.

Utgoff, P. E. 1994. An improved algorithm for incremental induction of decision trees. Machine Learning: Proceedings of the Eleventh International Conference, pp. 318-325. New Brunswick, NJ: Morgan Kaufmann.

Winston, P. H. 1984. Artificial intelligence. Reading, MA: Addison-Wesley. 